\title{
Comparison of findings using ultrasonography and cystoscopy in urogenital schistosomiasis in a public health centre in rural Angola
}

\author{
J Santos,,${ }^{1,2,9} \mathrm{MD} ;$ J Chaves, ${ }^{1,2,9} \mathrm{MD}, \mathrm{PhD} ; \mathbf{H}$ Araújo, ${ }^{2,9} \mathrm{MD} ; \mathbf{N}$ Vale, ${ }^{3}$ ChemD, $\mathrm{PhD} ; \mathbf{J}$ M Costa ${ }^{4} \mathrm{PhD} ; \mathbf{P}$ J Brindley, ${ }^{5} \mathrm{PhD}$; \\ C Lopes, ${ }^{6} \mathrm{MD}$, PhD; J Naples, ${ }^{7} \mathrm{MD}$, PhD; C Shiff, ${ }^{7} \mathrm{MD}$, PhD; J Dupret ${ }^{8} \mathrm{MD}$; L L Santos, ${ }^{6} \mathrm{MD}, \mathrm{PhD}$ \\ ${ }^{1}$ Urology Unit, Americo Boavida Hospital, Luanda, Angola \\ ${ }^{2}$ Urology Unit, Sagrada Esperança Clinic, Luanda, Angola \\ ${ }^{3}$ Centro de Investigação em Química da Universidade do Porto (CIQUP), Chemistry and Biochemistry Department, Faculty of Sciences, \\ University of Porto, Porto, Portugal \\ ${ }^{4}$ Centre for the Study of Animal Science, University of Porto, Portugal \\ ${ }^{5}$ Department of Microbiology, Immunology and Tropical Medicine, School of Medicine and Health Sciences, George Washington \\ University, Washington, DC, USA \\ ${ }^{6}$ Experimental Pathology and Therapeutics Group, Research Centre of the Portuguese Institute of Oncology, Porto, Portugal, and Oncocir - \\ Education and Care in Oncology and National Cancer Centre, Angola \\ ${ }^{7}$ Johns Hopkins Bloomberg School of Public Health, Baltimore, MD, USA \\ ${ }^{8}$ Ministry of Health, Angola \\ ${ }^{9}$ Agostinho Neto University, Luanda, Angola
}

Corresponding author: L L Santos (llarasantos@gmail.com)

Background. Schistosomiasis is a chronic disease caused by infection with parasitic worms of the genus Schistosoma. In sub-Saharan Africa, infections with S. haematobium are most common. Cystoscopic examination (CE) has been accepted as the gold-standard test for detecting the late manifestations of schistosomiasis, including urothelial cancer of the bladder. However, this procedure is invasive and 10 - $40 \%$ of tumours may remain undetected. A non-invasive examination and a new generation of biomarkers are needed for better monitoring of the disease. Objective. To assess the usefulness of ultrasound (US) scans for monitoring of structural urinary tract disease by local public health services in areas of Angola in which urogenital schistosomiasis is endemic.

Methods. A cohort of $80 \mathrm{~S}$. haematobium-infected patients was selected in order to compare changes in the bladder wall detected by US with those observed on CE.

Results. There was a notable correlation between the findings observed on CE and US. Patients with lesions of the bladder mucosa such as neoplasms, ulcers or granulomas detected by CE also had changes in bladder wall thickness on US. The results support increased use of portable US machines for non-invasive examination of the bladder by local general practitioners.

Conclusion. US examination should be an integral part of the investigation of haematuria and used in all S. haematobium control programmes. General practitioners may find it useful for more accurate diagnosis of haematuria and to identify bladder wall alterations in both adults and children in schistosomiasis-endemic regions.

S Afr Med J 2015;105(4):312-315. DOI:10.7196/SAMJ.8564

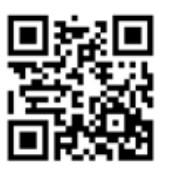

Anthropophilic species of Schistosoma currently infect more than 200 million people in 77 countries in the endemic areas of Africa, the Caribbean, Central America, South America, East Asia and the Middle East. ${ }^{[1]}$ More than $90 \%$ of these cases occur in Africa, and of these approximately two-thirds are caused by $S$. haematobium. ${ }^{[2]}$ Recently King ${ }^{[3]}$ suggested that the number of cases of $S$. haematobium infection may be greater than was previously believed. If this is confirmed, urogenital schistosomiasis may represent the most common infection in sub-Saharan Africa. ${ }^{[3]}$

Many infections with S. haematobium result in minimal symptoms or cause haematuria, dysuria, anaemia and inflammation of the urinary tract. However, a significant proportion of infected patients, ranging from $25 \%$ to $50 \%$, experience moderate to severe morbidity. Late manifestations include kidney dysfunction, ureteric obstruction and squamous cell carcinoma of the bladder. Cystoscopic examination (CE) is accepted as the gold standard for the detection of disorders of chronic schistosomiasis. ${ }^{[4]}$ However, this procedure is invasive and $10-40 \%$ of malignancies may be undetected. Microscopic examination of the urine remains the gold-standard test for the diagnosis of urogenital schistosomiasis, although it has some limitations. Detection of specific antibodies and/or antigens by serological assays can be useful in specific circumstances in endemic areas, but their application is limited ${ }^{[5]}$ beyond diagnosis of the disease in travellers from non-endemic areas. ${ }^{[6]}$ Schistosomiasis can be controlled with safe and effective drug therapy. Other components of management include the provision of potable water, adequate sanitation, hygiene education and snail control. ${ }^{[6-7]}$

In a resource-poor setting with numerous limitations, what can general practitioners, other healthcare professionals and local health services do for populations in their care in regions endemic for schistosomiasis? Lesions caused by infection with S. haematobium can be detected readily on ultrasound (US) examination. King ${ }^{[3]}$ reported that US-based population surveys enabled effective assessment of reversibility of structural changes in a range of age groups, and provided data assisting choice of treatment protocols.

The present study assessed the performance of US detection of bladder wall changes in a group of patients in a schistosomiasisendemic area (Pita, Angola; Fig. 1) referred to a public clinic with 
nonspecific haematuria, comparing and contrasting changes in the bladder wall detected by US with those observed at CE. To our knowledge, this is the first time such an assessment has been done.

\section{Methods}

\section{Study sites and participants}

Ethical approval for the study was granted by the ethics committee of Agostinho Neto University, Luanda, Angola. Parental consent in the case of children and consent from participating adults was obtained.

Eighty people were recruited into the study between December 2011 and September 2012. Of these, 70 were consecutive patients with macroscopic haematuria who attended a rural public clinic (PitaMuxima) in Luanda Province (municipality of Quissama). An additional 10 were admitted to the Department of Urology at Américo Boavida Hospital (ABH), Luanda, with haematuria and suspected bladder cancer. All participants had bladder US scans with subsequent $\mathrm{CE}$ and mucosal biopsy.

\section{Urine}

Urine samples were collected from the 80 patients. They were tested for haematuria using reagent strips (Hemastix, Bayer, UK). Parasitological examination for detection of $S$. haematobium eggs was done by filtering a $10 \mathrm{ml}$ aliquot through a polycarbonate filter with a pore size of $12 \mu \mathrm{m}$ diameter (Millipore, UK). The slides were examined at $\times 100$ under a compound light microscope, according to World Health Organization $(\mathrm{WHO})$ guidelines ${ }^{[8]}$

\section{Ultrasound examination}

Fluid was given to each patient 30 minutes to 1 hour before US examination to ensure adequate filling of the bladder to assess its shape and any wall irregularity. A portable US apparatus (Siemens portable ultrasound with a 3.5 MHz curvilinear probe, WA 980297002 , USA) was used. The scans were done by medically qualified persons, particular attention being paid to abnormalities of bladder shape, irregularities or thickening of the bladder wall, discernible masses and presence of polyps, calcification and/ or hydronephrosis. Findings were recorded photographically. WHO criteria were used to classify any bladder damage detected. ${ }^{[9]}$

\section{Cystoscopic examination}

Patients underwent CE in the Department of Urology at $\mathrm{ABH}$. CE was performed after the US scan, and particular attention was given to major bladder mucosa alterations such as ulcers, schistosome eggs, granulomas and tumours (14 patients had schistosome eggs and other mucosal changes). A Karl
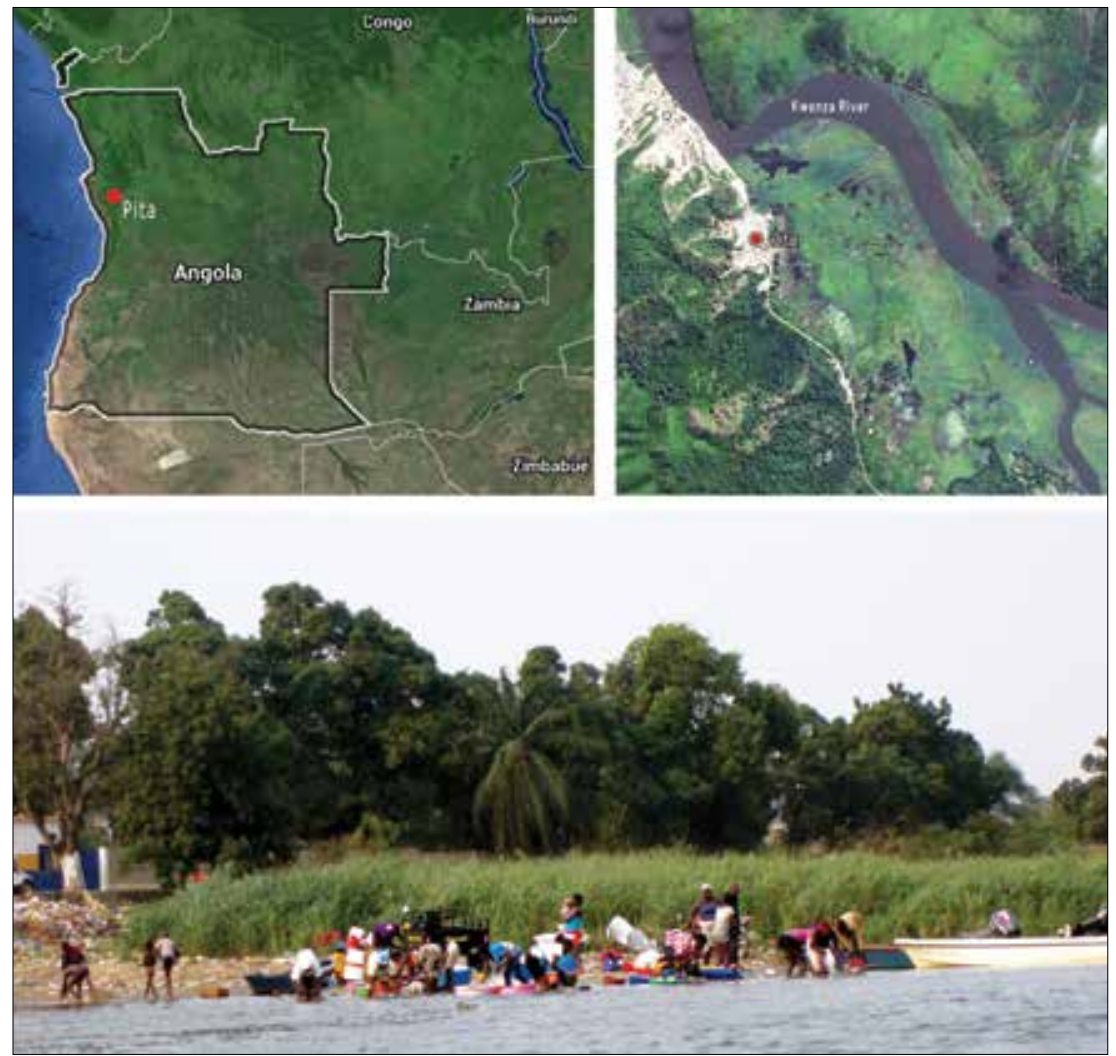

Fig. 1. Pita, Luanda Province, Angola.

Storz cystoscope, 20-Fr (adults) and 11.5-Fr (children), with a $30^{\circ}$ eyepiece was used.

\section{Treatment}

Prazinquantel $40 \mathrm{mg} / \mathrm{kg}$ was offered to patients diagnosed with schistosomiasis, according to WHO guidelines. ${ }^{[10]}$ Those who were diagnosed with bladder cancer were admitted to the urology service of $\mathrm{ABH}$ for specific treatment.

\section{Statistical analysis}

Statistical analysis was performed using SPSS 17 statistics software (SPSS Inc. for Windows). Demographic, bladder US and cystoscopy data were recorded. $\chi^{2}$ analysis using Fisher's exact test (two-tailed) was performed for categorical data, with a $p$-value of $\leq 0.05$ considered statistically significant.

\section{Results}

The median age of the patients was 41 years (range 3 - 75); 62 (77.5\%) were males. All had macroscopic haematuria, and the reagent strips were positive for the presence of blood. A parasite egg count was positive in 36 patients (45.0\%). All the paediatric patients were treated for the first time during this study. Bladder wall irregularities detected by US are described in Table 1. In brief, 60 (75.0\%) of the participants had distortion of the bladder shape and 29 (36.3\%) had bladder masses (Fig. 2). Major findings on CE are set out in Tables 1 and 2.

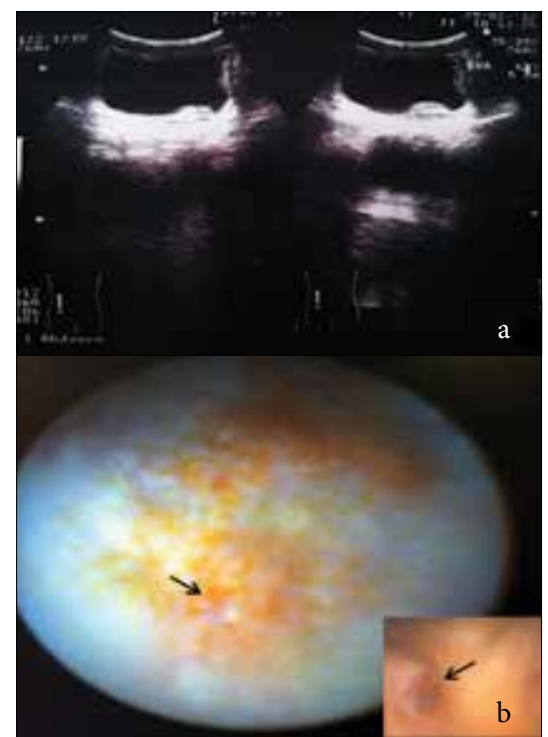

Fig. 2. (a) US bladder wall irregularities and (b) appearance on CE in a patient with haematuria (arrows = granuloma).

Here, 54 patients $(67.5 \%)$ had only schistosome eggs, granulomas being present in 14 (17.5\%). Neoplasms were seen in 10 patients $(12.5 \%)$ and ulcerations in 2 (2.5\%), both of whom were $<19$ years of age. The median age of patients with tumours was 51 years (range 10 - 68).

There was a notable correlation between the findings on CE and US. Patients with lesions in the bladder mucosa such as neoplasms, ulcers or granulomas detected by 
Table 1. Gender, presence of haematuria, bladder US and CE features and findings on examination of the urine

\begin{tabular}{|c|c|}
\hline Variables & $\begin{array}{l}\text { Patients } \\
n(\%)\end{array}$ \\
\hline \multicolumn{2}{|l|}{ Gender } \\
\hline Female & $18(22.5)$ \\
\hline Male & $62(77.5)$ \\
\hline Haematuria & $80(100.0)$ \\
\hline \multicolumn{2}{|l|}{ US bladder shape } \\
\hline Normal & $20(25.0)$ \\
\hline Abnormal & $60(75.0)$ \\
\hline \multicolumn{2}{|c|}{ US bladder wall irregularities } \\
\hline$<5 \mathrm{~mm}$ normal & $1(1.3)$ \\
\hline$\geq 5 \mathrm{~mm}$ focal & $40(50.0)$ \\
\hline $\begin{array}{l}\geq 5 \mathrm{~mm} \text { multifocal or } \\
\text { diffuse }\end{array}$ & $39(48.7)$ \\
\hline \multicolumn{2}{|l|}{ US discernible masses } \\
\hline None & $51(63.7)$ \\
\hline Single & $13(16.3)$ \\
\hline Multiple & $16(20.0)$ \\
\hline \multicolumn{2}{|c|}{ US presence of pseudopolyps } \\
\hline Yes & $6(7.5)$ \\
\hline No & $74(92.5)$ \\
\hline \multicolumn{2}{|l|}{ US calcification } \\
\hline Yes & $75(93.7)$ \\
\hline No & $5(6.3)$ \\
\hline \multicolumn{2}{|l|}{ Hydronephrosis } \\
\hline Yes & $3(3.7)$ \\
\hline No & $77(96.3)$ \\
\hline \multicolumn{2}{|l|}{$\mathrm{CE}$} \\
\hline S. haematobium eggs & $54(67.5)$ \\
\hline Granuloma & $14(17.5)$ \\
\hline Ulcer & $2(2.5)$ \\
\hline Neoplasm & $10(12.5)$ \\
\hline \multicolumn{2}{|l|}{ Urine } \\
\hline S. haematobium eggs & $36(45.0)$ \\
\hline
\end{tabular}

$\mathrm{CE}$ also had changes in bladder thickness on US. Only one patient in whom CE showed the presence of eggs in the mucosa had a normal US scan (Table 3). Residual urine was observed in 17 patients (21.3\%), and bilateral hydronephrosis was found in 3 (3.8\%). One patient had an associated kidney neoplasm.

The outcomes achieved by the two diagnostic approaches supported an acceptable correlation for the detection of bladder changes by US and CE. While bladder wall distortion or bladder masses in urogenital schistosomiasis were detected by US, it was not possible to obtain meaningful information about bladder thickness (Fig. 3).

Table 2. Dominant features on bladder CE according to age group

\begin{tabular}{llllll}
\hline & \multicolumn{5}{c}{ Patients, $\boldsymbol{n}$} \\
\cline { 2 - 5 } Age group (years) & S. haematobium eggs & Granuloma & Ulcer & Neoplasm & Total \\
\hline$\leq 19$ & 4 & 8 & 2 & 1 & 15 \\
$20-29$ & 12 & 1 & 0 & 1 & 14 \\
$30-39$ & 4 & 3 & 0 & 1 & 8 \\
$40-49$ & 18 & 1 & 0 & 1 & 20 \\
$50-59$ & 8 & 0 & 0 & 4 & 12 \\
$60-69$ & 6 & 0 & 0 & 2 & 8 \\
$\geq 70$ & 2 & 1 & 0 & 0 & 3 \\
Total & 54 & 14 & 2 & 10 & 80
\end{tabular}

Table 3. Dominant features on bladder CE and irregularities noted on bladder US

\begin{tabular}{llllll}
\hline \multirow{2}{*}{ Cystoscopy features } & US bladder wall irregularities & None & Single & Multiple & Total, $\boldsymbol{n}$ \\
\cline { 2 - 5 } S. haematobium eggs & $<5 \mathrm{~mm}-$ normal & 1 & - & - & 1 \\
& $\geq 5 \mathrm{~mm}$ focal & 29 & 3 & - & 32 \\
Granuloma & $\geq 5 \mathrm{~mm}$ multifocal or diffuse & 18 & 2 & 1 & 21 \\
& $<5 \mathrm{~mm}-$ normal & - & - & - & - \\
Ulcer & $\geq 5 \mathrm{~mm}$ focal & 1 & - & 1 & 2 \\
& $\geq 5 \mathrm{~mm}$ multifocal or diffuse & 1 & 2 & 9 & 12 \\
Neoplasm & $<5 \mathrm{~mm}$ - normal & - & - & - & - \\
& $\geq 5 \mathrm{~mm}$ focal & 1 & - & - & 1 \\
& $\geq 5 \mathrm{~mm}$ multifocal or diffuse & 1 & - & - & 1 \\
& $\geq 5 \mathrm{~mm}$ focal & - & 5 & - & 5 \\
& $\geq 5 \mathrm{~mm}$ multifocal or diffuse & - & - & 5 & 5
\end{tabular}

\section{Discussion}

US is suitable for monitoring schistosomiasis-related pathology of the urinary tract, and is particularly useful in assessing its evolution after therapy. Bladder alterations observed by US have been associated with known biomarkers of bladder cancer in adults with chronic $S$. haematobium infection. ${ }^{[11]}$ The importance of US examination in monitoring structural disorders in urogenital schistosomiasis has long been established. US provides clear evidence of ureteric wall abnormalities causing strictures and ureterocelelike lesions of the ureteric ostia, and demonstrates resolution of bladder lesions after treatment, including in paediatric patients. ${ }^{[12]}$

A recent report indicates that general practitioners can learn to carry out a simple US examination for accurate diagnosis and followup of S. haematobium infection-related bladder lesions within a few sessions. ${ }^{[13]}$ The aim of the present study was to verify the reproducibility of the bladder changes detected by US and bladder mucosa lesions observed by $\mathrm{CE}$ in order to teach general doctors to perform bladder US as an additional tool in the diagnosis of haematuria in areas endemic for S. haematobium.
Our results support other observations on $S$. haematobium-infected patients $>30$ years of age, in whom severe bladder damage was evident on US examination but no $S$. haematobium eggs were present in their urine samples. ${ }^{[14]}$ The optimal imaging modality for patients with haematuria remains controversial, particularly given developments in computed tomography urography and magnetic resonance urography ${ }^{[15]}$ However, the European Society of Urogenital Radiology guidelines for the investigation of painless haematuria recommend the use of US. ${ }^{[16]}$

Our results confirm that in patients with haematuria, the changes seen on US examination of the bladder correlated significantly with the presence of eggs of $S$. haematobium, ulcers or granulomas in the bladder mucosa observed at CE. We consider that a training programme for general practitioners in US for this purpose would be valuable in developing countries. Portable US devices are also useful for the evaluation of breast, gynaecological and urological pathology, and should be among the tools employed for routine examinations by the general doctor in resource-poor settings. ${ }^{[17]}$ 


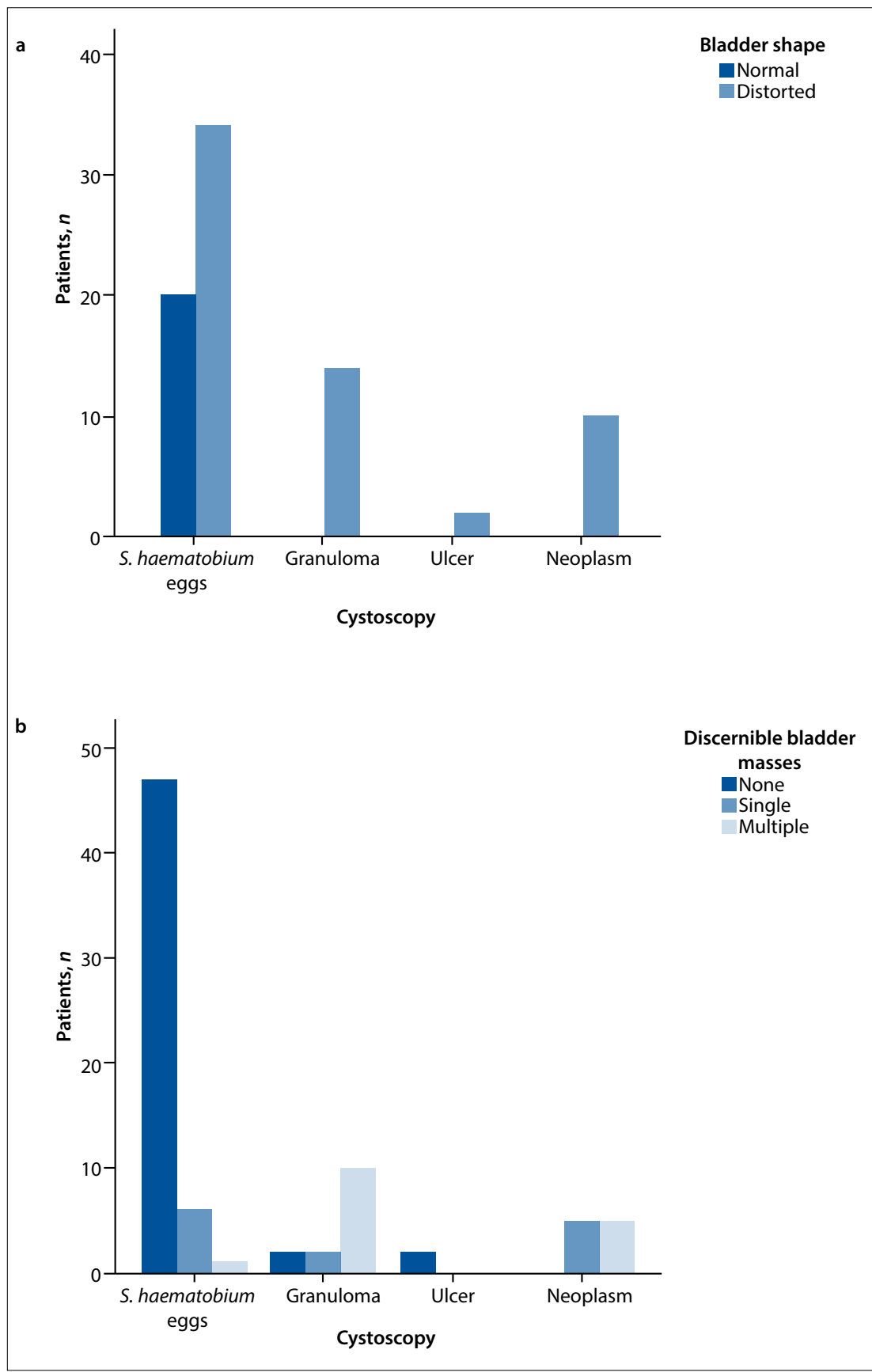

Fig. 3. Correlation between CE features and bladder changes on US: (a) bladder shape on US ( $\mathrm{p}=0.005)$; (b) masses detected on US ( $\mathrm{p}=0.0001)$.

Infection with $S$. haematobium has been detected in $64.8 \%$ of adults examined in the north of Angola. ${ }^{[18]}$ At the 2014 International Congress of Angolan Physicians in Luanda, Pintar et al. ${ }^{[19]}$ presented their finding that $83 \%$ of 149 children with S. haematobium infection had bladder wall changes that were evident on US.

Angola has a programme of prevention, control and elimination of neglected diseases such as schistosomiasis that includes the training of general practitioners in US to help achieve early diagnosis of urogenital schistosomiasis and to monitor the results of chemotherapy. The introduction of
$\mathrm{ABH}$ and Sagrada Esperança Clinic, and the Dean of the Faculty of Medicine of Agostinho Neto University, Prof. Miguel Betencourt Mateus. Author contributions. JS and LLS conceived and designed the experiments, JS, JC and HA performed the experiments, JS, CL, JMC and LLS analysed the data, and JS, CS, JD, JN, NV, CL, PJB, JMC and LLS wrote the paper.

\section{References}

1. Mostafa MH, Sheweita SA, O'Connor PJ. Relationship between schistosomiasis and bladder cancer. Clin Microbiol Rev 1999;12(1):97-111.

2. Brindley PJ, Hotez P. Break out: Urogenital schistosomiasis and Schistosoma haematobium in the post-genomic era. PLoS Negl Trop Dis 2013;7(3):e196. [http://dx.doi.org/10.1371/journal.pntd.0001961]

3. King $\mathrm{CH}$. Ultrasound monitoring of structural urinary tract disease in Schistosoma haematobium infection. Mem Inst Oswaldo Cruz 2002;97(1):149-152. [http://dx.doi.org/10.1590/ S0074-02762002000900028]

4. Devidas A, Lamothe F, Develoux M, Gakwaya I, Ravisse P, Sellin B. [Morbidity due to bilharziasis caused by S. haematobium: Relationship between the bladder lesions observed by ultrasonography and the cystoscopic and anatomo-pathologic lesions]. Acta Trop 1988;45(3):277-287.

5. Nausch N, Dawson EM, Midzi N, Mduluza T, Mutapi F, Doenhoff MI. Field evaluation of a new antibody-based diagnostic for S. Field evaluation of a new antibody-based diagnostic for Schistosoma haematobium and S. mansoni at the point-of-care in
northeast Zimbabwe. BMC Infect Dis 2014;14(3):165-173. [http:// northeast Zimbabwe. BMC Infect Dis 2014

6. Pardo J, Carranza C, Turrientes MC, et al. Utility of Schistosoma bovis

6. Pardo), Carranza C, Turrientes MC, et al. Utility of Schistosoma bovis
adult worm antigens for diagnosis of human schistosomiasis by enzyme-linked immunosorbent assay and electroimmunotransfer blot techniques. Clin Diagn Lab Immunol 2004;11(6):1165-1170. [http://dx.doi.org/10.1128/CDLI.11.6.1165-1170.2004]

7. Engles D, Chitsulo L, Montresor A, Savioli L. The global epidemiological situation of schistosomiasis and new approaches to control and research. Acta Trop 2002;82(2):139-146.

8. Montresor A, Crompton DWT, Bundy DAP, Hall A, Savoli L. Guidelines for the Evaluation of Soil-transmitted Helminthiasis and Schistosomaisis at Community Level: A Guide for Managers of Control Programmes. Geneva: WHO, 1998.

9. Richter J, Hatz C, Campagne G, Bergquist NR, Jenkins JM.

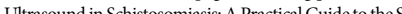
Utrasound hn Schistosoniasis. A Practical Guide to he Standardized related Morbidity. Geneva: World Health Organization, 2000.

10. World Health Organization, Prevention and control of World Health Organization, Prevention and control of
schistosomiasis and soil-transmitted helminthiasis. WHO schistosomiasis and soil-transmitted helminthiasis.
Technical Reports Series No. 912. Geneva: WHO, 2002.

Technical Reports Series No. 912 . Geneva: WHO, 2002.
Shiff C, Veltri R, Naples J, et al. Ultrasound verification of bladder 11. Shiff C, Veltri R, Naples J, et al. Ultrasound verification of bladder
damage is associated with known biomarkers of bladder cancer in adults chronically infected with Schistosoma haematobium in Ghana. Trans R Soc Trop Med Hyg 2006;100(9):847-854. [http:// dx.doi.org/10.1016/j.trstmh.2005.10.010]

12. Kardorff R, Traoré M, Doehring-Schwerdtfeger E, Vester U, Ehrich JH. Ultrasonography of ureteric abnormalities induced by Schistosoma haematobium infection before and after praziquantel treatment. Br J Urol 1994;74(6):703-709.

13. Bonnard P, Boutouaba S, Diakhate I, Seck M, Dompnier JP, Riveau G. Learning curve of vesico-urinary ultrasonography in
Rinter Riveau G. Learning curve of vesico-urinary ultrasonography in
Schistosoma haematobium infection with WHO practical guide: A Schistosoma haematobium infection with WHO practical guide: A
'simple to learn' examination. Am J Trop Med Hyg 2011;85(6):1071'simple to learn' examination. Am J Trop Med Hyg 2011; 856

1074. [http://dx.doi.org/10.4269/ajtmh.2011.11-0282] 14. Shiff C. The importance of definitive diagnosis in chronic
schistosomiasis, with reference to Schistosoma haematobium. J Parasitol schistosomiasis, with reference to Schistosoma haematobium. J Parasit
Res 2012;2012:761269. [http://dx.doi.org/10.1155/2012/761269]

15. O'Connor OJ, McSweeney SE, Maher MM. Imaging of hematuria. Radiol Clin North Am 2008;46 (1):113-132. [http:// dx.doi.org/10.1016/j.rcl.2008.01.007]

16. Van der Molen AJ, Cowan NC, Mueller-Lisse UG, NolteErnsting CC, Takahashi S, Cohan RH, CT Urography Working Group of the European Society of Urogenital Radiology (ESUR). GT urography: Definition, indications and techniques CT urography: Definition, indications and techniques:

17. She 1 , Long 7. Shetty M, Longatto-Filho A. Early detection of breast, cervical, ovarian and endometrial cancers in low resource countries: An integrated approach. Indian J Surg Oncol 20182 [http://dx.doi.org/10.1007/s13193-011-0082-6]

18. Sousa-Figueiredo JC, Gamboa D, Pedro JM, et al. Epidemiology of malaria, schistosomiasis, geohelminths, anemia and malnutrition in the context of a demographic surveillance system in northern Angola. PLoS One 2012;7(4):e33189. [http:// dx.doi.org/10.1371/journal.pone.0033189]

19. Pintar Z, Bocanegra C, Molina I, Moreno M, Salvador F, Serres X. Ultrasonographic findings in Schistosoma haematobium infection in paediatrics (Poster). Presented at the International Congress of the Order of Physicians, Luanda, Angola, 24-25 January 2014. 20. Shiff C, Naples JM, Isharwal S, Bosompem KM, Veltri RW. Noninvasive methods to detect schistosome-based bladder cancer: Is the

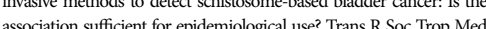
association sufficient for epidemiological use? Trans R Soc Trop Med

Acknowledgments. The authors thank the team of Pita Public Clinic, the urological services of 